\title{
Ladislav Bican
}

Notes on purities

Czechoslovak Mathematical Journal, Vol. 22 (1972), No. 4, 525-534

Persistent URL: http://dml.cz/dmlcz/101124

\section{Terms of use:}

(C) Institute of Mathematics AS CR, 1972

Institute of Mathematics of the Czech Academy of Sciences provides access to digitized documents strictly for personal use. Each copy of any part of this document must contain these Terms of use.

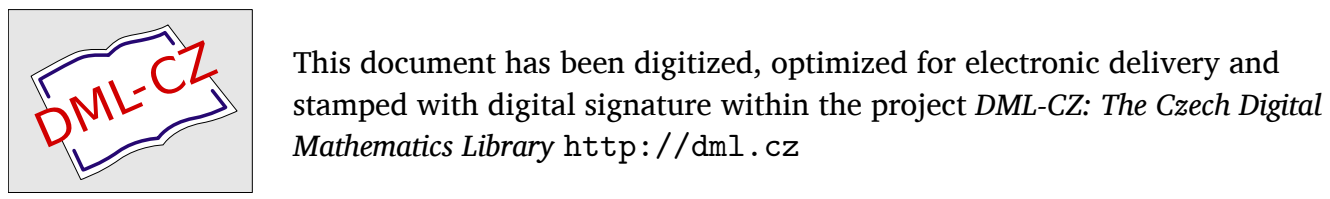




\title{
NOTES ON PURITIES
}

\author{
Ladislav Bican, Praha
}

(Received February 25, 1971)

Throughout this paper, the word "module" always means a unitary $\Lambda$-module where $\Lambda$ is an associative ring with unity. The basic definitions are given in [5] or [1].

\section{PROJECTIVELY CLOSED PURITIES}

The definitions and notations are given in [5] or [1] and therefore we do not repeat them. The composition of the homomorphisms $\varphi: A \rightarrow B, \psi: B \rightarrow C$ is denoted by $\psi \varphi$.

We start with the following

Lemma 1. Let $U$ be a submodule of a free module $F, 0 \rightarrow U \stackrel{\chi}{\rightarrow} F \stackrel{\eta}{\rightarrow} P \rightarrow 0$ an exact sequence where $\chi$ is the canonical embedding and $i: A \rightarrow B$ a monomorphism. Then $P$ is co-projective with respect to $i$ if and only if $i \in \mathfrak{H}_{F U}$.

Proof. This proof is essentially the same as that of Lemma 1 in [5] and therefore we omit it.

From Theorem 1 and Lemma 3 from [5] it follows that the three following properties of a purity $\omega$ are equivalent:

a) $\omega$ is projectively closed,

b) $\omega$ is a $\Gamma$-purity for some class $\Gamma$,

c) $\omega$ is of the form $\omega^{\mathfrak{M}}$ for some class of modules $\mathfrak{M}$.

Definition 1. Let $\omega$ be a projectively closed purity. An arbitrary class $\Gamma$ of couples $(F, U)$ where $U$ is a submodule of a free module $F$ satisfying $\mathfrak{H}_{\omega}=\mathfrak{H}_{\Gamma}$ will be called a basis of $\omega$. Similarly, an arbitrary class $\mathfrak{M}$ of modules satisfying $\omega=\omega^{\mathfrak{M}}$ will be called a $\mathfrak{P}$-basis of $\omega$.

The following simple lemma will be useful in the sequel. 
Lemma 2. If $\Gamma=\{(F, U)\}$ is a basis of a projectively closed purity $\omega$ then the class $\mathfrak{M}=\{P, P=F / U,(F, U) \in \Gamma\}$ is a $\mathfrak{P}$-basis of $\omega$. Conversely, if the class $\mathfrak{M}$ is a $\mathfrak{P}$-basis of a projectively closed purity $\omega$ then taking to any $P \in \mathfrak{M}$ an exact sequence $0 \rightarrow U \stackrel{\chi}{\rightarrow} F \stackrel{\eta}{\rightarrow} P \rightarrow 0$ where $U$ is a submodule of a free module $F$ and $\chi$ is the canonical embedding we obtain that the class $\Gamma$ of all such couples $(F, U)$ is a basis of $\omega$.

Proof follows easily from Lemma 1.

Recall that a family $A_{\alpha}, \alpha \in \Omega$ of submodules of $A$ is called a covering of $A$ if $A_{\alpha}$, $\alpha \in \Omega$ generate $A$. Further, a module $A$ is called compact if its any countable covering has a finite subcovering.

Theorem 1. If a projectively closed purity $\omega$ has a $\mathfrak{P}$-basis $\mathfrak{M}$ such that any module from $\mathfrak{M}$ is compact then the class $\mathfrak{G}_{\omega}$ is closed under taking direct sums.

Proof. Let $0 \rightarrow A_{\alpha} \stackrel{i_{\alpha}}{\rightarrow} B_{\alpha} \stackrel{\pi_{\alpha}}{\rightarrow} C_{\alpha} \rightarrow 0, \alpha \in \Omega$ be any set of short exact sequences with $i_{\alpha} \in \mathfrak{H}_{\omega}$. Let us put $A=\sum_{\alpha \in \Omega} A_{\alpha}, B=\dot{\sum}_{\alpha \in \Omega} B_{\alpha}, C=\sum_{\alpha \in \Omega} C_{\alpha}, i=\sum_{\alpha \in \Omega} i_{\alpha}, \pi=\sum_{\alpha \in \Omega} \pi_{\alpha}$ and let $P \in \mathfrak{M}$ be an arbitrary module. Then the sequence $0 \rightarrow A \stackrel{i}{\rightarrow} B \stackrel{\pi}{\rightarrow} C \rightarrow 0$ is exact and the compactness of $P$ guarantees that the image of any homomorphism $f: P \rightarrow C$ is contained in the direct sum of a finite number of $C_{\alpha}^{\prime}$ s (see [1], p. 47). The class $\mathfrak{H}_{\omega}$ is closed under taking finite direct sums (see $(1,1)$ in [1]) which guarantees the existence of the homomorphism $\varphi: P \rightarrow B$ with $\pi \varphi=f$. Therefore $i \in \mathfrak{H}_{\omega}$ and the proof is complete.

Theorem 2. If a projectively closed purity $\omega$ has a $\mathfrak{P}$-basis $\mathfrak{M}$ such that any module from $\mathfrak{M}$ is compact then the direct sum $A=\sum_{\alpha \in \Omega} A_{\alpha}$ is $\omega$-flat if and only if any $A_{\alpha}$, $\alpha \in \Omega$ is $\omega$-flat.

Proof. Recall that a module $A$ is $\omega$-flat if $i \in \mathfrak{H}_{\omega}$ for any exact sequence $0 \rightarrow K \stackrel{i}{\rightarrow}$ $\stackrel{i}{\rightarrow} B \rightarrow A \rightarrow 0$. If $A$ is $\omega$-flat then any $A_{\alpha}, \alpha \in \Omega$ is $\omega$-flat by $(1,15)$ from [1]. Conversely, let us suppose that any $A_{\alpha}, \alpha \in \Omega$ is $\omega$-flat. Taking for any $A_{\alpha}, \alpha \in \Omega$ an exact sequence $0 \rightarrow U_{\alpha} \stackrel{i_{\alpha}}{\rightarrow} F_{\alpha} \rightarrow A_{\alpha} \rightarrow 0$ with a free module $F_{\alpha}$ we have $i_{\alpha} \in \mathfrak{H}_{\omega}$. Then the sequence $0 \rightarrow \sum_{\alpha \in \Omega} U_{\alpha} \stackrel{\sum_{\alpha \in \Omega} i_{\alpha}}{\longrightarrow} \sum_{\alpha \in \Omega} F_{\alpha} \rightarrow A \rightarrow 0$ is exact and $\sum_{\alpha \in \Omega} i_{\alpha} \in \mathfrak{H}_{\omega}$ by Theorem 1 . Hence $A$ is $\omega$-flat by $(1,12)$ from [1].

Theorem 3. If a projectively closed purity $\omega$ has a basis $\Gamma$ where $\Gamma$ is a set then there exists a free module $F$ and its submodule $U$ such that $\mathfrak{H}_{\omega}=\mathfrak{H}_{F U}$.

Proof. By Lemma 2, $\omega$ has a $\mathfrak{B}$-basis $\mathfrak{M}$ where $\mathfrak{M}$ is a set. By $(1,5)$ from [1] the module $P=\sum_{P^{\prime} \in \mathfrak{M}} P^{\prime}$ is $\omega$-projective, $P \in \mathfrak{P}_{\omega}$, so that $\mathfrak{H}_{\omega} \subseteq \mathfrak{H}^{\{P\}}$. Conversely, again 
by $(1,5)$ from $[1]$ we have $\mathfrak{M} \subseteq \mathfrak{P}_{\omega}\{P\}$ hence $\mathfrak{H}^{\{P\}} \subseteq \mathfrak{H}_{\omega}$ and Lemma 1 completes the proof.

Theorem 4. If a projectively closed purity $\omega$ has a $\mathfrak{P}$-basis $\mathfrak{M}$ (or a basis $\Gamma$ ) which is a set, then the purity $\omega$ is projective.

Proof. In view of Lemma 2 and Theorem 3 we can assume that the module $P^{\prime}$ is a $\mathfrak{P}$-basis of $\omega$. Let $A$ be an arbitrary module and $\pi^{\prime}: F \rightarrow A$ an epimorphism of some free module $F$ onto $A$. By $(1,5)$ from [1] the module $P=F+\sum_{f \in \operatorname{Hom}\left(P^{\prime}, A\right)} P_{f}^{\prime}$ where $P_{f}^{\prime}=P^{\prime}$ for any $f \in \operatorname{Hom}\left(P^{\prime}, A\right)$ is $\omega$-projective. For an element $\left(q,\left(p_{f}^{\prime}\right)\right) \in P$, $q \in F, p_{f}^{\prime} \in P_{f}^{\prime}$, let us put $\pi\left(\left(q,\left(p_{f}^{\prime}\right)\right)\right)=\pi^{\prime}(q)+\sum_{f \in \operatorname{Hom}\left(P^{\prime}, A\right)} f\left(p_{f}^{\prime}\right)$ (this can be made since only a finite number of $p_{f}^{\prime}$ 's is non-zero). Here $\pi: P \rightarrow A$ is an epimorphism since $\pi^{\prime}$ is. If we denote $K=\operatorname{Ker} \pi$ and $i$ the corresponding canonical embedding we get an exact sequence $0 \rightarrow K \stackrel{i}{\rightarrow} P \stackrel{\pi}{\rightarrow} A \rightarrow 0$. It remains to show that $i \in \mathfrak{H}_{\omega}$. However, taking $f \in \operatorname{Hom}\left(P^{\prime}, A\right)$ arbitrarily and denoting by $L_{f}$ the canonical embedding of $P^{\prime}=P_{f}^{\prime}$ into $P$ we obviously have $\pi \iota_{f}=f$ and the proof is complete.

Remark. We have just proved something more, namely: If a projectively closed purity $\omega$ has a set as a $\mathfrak{P}$-basis then there exists a module $P^{\prime} \in \mathfrak{P}_{\omega}$ such that to any module $A$ there is an exact sequence $0 \rightarrow K \stackrel{i}{\rightarrow} P \rightarrow A \rightarrow 0$ with $i \in \mathfrak{H}_{\omega}$ and $P=$ $=F+\sum_{f \in \operatorname{Hom}\left(P^{\prime}, A\right)} P^{\prime}$ where $F$ is free and $P_{f}^{\prime}=P^{\prime}$ for any $f \in \operatorname{Hom}\left(P^{\prime}, A\right)$.

Theorem 5. The following two conditions are logically equivalent:

a) Any projectively closed purity has a set as a $\mathfrak{P}$-basis,

b) there exists a cardinal number $m$ such that any module of power at least $m$ is a direct sum of modules of powers less than $m$.

Proof. First, let us show that a) $\Rightarrow$ b). For this purpose, let us assume the purity $\omega$ to have the class of all modules as a $\mathfrak{P}$-basis. By hypothesis, Theorem 3 and Lemma 2, there exists a module $P^{\prime}$ which is a $\mathfrak{P}$-basis of $\omega$. Let $m$ be the first uncountable cardinal greater than $\left.\max \left(\left|P^{\prime}\right|,|\Lambda|\right)^{1}\right)$. By the remark preceding this theorem, to any module $A$ of power at least $m$ there exists an exact sequence $0 \rightarrow K \stackrel{i}{\rightarrow} P \rightarrow A \rightarrow 0$ with $i \in \mathfrak{H}_{\omega}$ and $P=F+\sum_{f \in \operatorname{Hom}\left(P^{\prime}, A\right)} P_{f}^{\prime}$ where $F$ is free and $P_{f}^{\prime}=P^{\prime}$ for any $f \in$ $\in \operatorname{Hom}\left(P^{\prime}, A\right)$. Hence $A$ is isomorphic to a direct summand of $P$ since $A \in \mathfrak{P}_{\omega}$ by hypothesis. Therefore $A$ is a direct sum of modules of powers less than $m$ owing to Theorem 4.3 from [4].

Conversely, let us suppose b) and let $\omega$ be any projectively closed purity. Let $\mathfrak{M}$ be the set of all pair-wise non-isomorphic modules from $\mathfrak{P}_{\omega}$ the powers of which are

1) $|M|$ denotes the power of the set $M$. 
less than $m$. Clearly, $\mathfrak{H}_{\omega} \subseteq \mathfrak{H}^{\mathfrak{M}}$. On the other hand, any module $P \in \mathfrak{P}_{\omega}$ of power at least $m$ is, by hypothesis, a direct sum $P=\sum_{\alpha \in \Omega} P_{\alpha}$ of modules of powers less than $m$. By (1.5) from [1] any $P_{\alpha}$ lies in $\mathfrak{P}_{\omega}$ and hence it is isomorphic to an element from $\mathfrak{M}$. Using (1.5) from [1] again, we get $P \in \mathfrak{P}_{\omega}^{\mathfrak{M}}$, hence $\mathfrak{P}_{\omega} \subseteq \mathfrak{P}_{\omega} \mathfrak{M}$ and $\mathfrak{H}^{\mathfrak{M}} \subseteq \mathfrak{H}_{\omega}$.

Definition 2. Let $\mathfrak{M}$ and $\mathfrak{N}$ be two sets of modules containing $\Lambda$. We say that $\mathfrak{N}$ depends on $\mathfrak{M}$ if any module from $\mathfrak{N}$ is isomorphic to a direct summand of a direct sum of modules from $\mathfrak{M}$. Further, we say that $\mathfrak{M}$ and $\mathfrak{N}$ are equivalent if $\mathfrak{M}$ depends on $\mathfrak{N}$ and conversely, $\mathfrak{N}$ depends on $\mathfrak{M}$.

Theorem 6. Let $\mathfrak{M}$ and $\mathfrak{N}$ be two sets of modules containing $\Lambda$. Then $\mathfrak{H}^{\mathfrak{M}} \subseteq \mathfrak{S}^{\mathfrak{M}}$ if and only if $\mathfrak{N}$ depends on $\mathfrak{M}$.

Particularly, $\mathfrak{H}^{\mathfrak{M}}=\mathfrak{H}^{\mathfrak{N}}$ if and only if $\mathfrak{M}$ and $\mathfrak{N}$ are equivalent.

Proof. The special assertion is a trivial consequence of the general one. First, let us suppose that $\mathfrak{H}^{\mathfrak{M}} \subseteq \mathfrak{H}^{\mathfrak{M}}$ and let $N \in \mathfrak{N}$ be an arbitrary module. By hypothesis, the proof of Theorem 3 and the remark after Theorem 4 there exists an exact sequence $0 \rightarrow K \stackrel{i}{\rightarrow} P \rightarrow N \rightarrow 0$ where $i \in \mathfrak{H}^{\mathfrak{M}}$ and $P$ is a direct sum of modules from $\mathfrak{M}$. Since $\mathfrak{H}^{\mathfrak{M}} \subseteq \mathfrak{H}^{\mathfrak{M}}, N$ is co-projective with respect to $i$ and therefore it is isomorphic to a direct summand of $P$. Hence $\mathfrak{N}$ depends on $\mathfrak{M}$.

Conversely, if $\mathfrak{N}$ depends on $\mathfrak{M}$ then $(1,5)$ from [1] yields $\mathfrak{N} \subseteq \mathfrak{P}_{\omega} \mathfrak{M}$ and therefore $\mathfrak{H}^{\mathfrak{M}} \subseteq \mathfrak{H}^{\mathfrak{M}}$.

Theorem 7. The intersection of any set of projective purities is a projective purity.

Proof. Let $\omega_{\alpha}, \alpha \in M$ be a set of projective purities and let us put $\omega=\bigcap_{\alpha \in M} \omega_{\alpha}$. It is clear that $i \in \mathfrak{Y}_{\omega}$ if and only if any module from $\bigcup_{\alpha \in M} \mathfrak{P}_{\omega_{\alpha}}$ is co-projective with respect to $i$ so that $\bigcup_{\alpha \in M} \mathfrak{P}_{\omega_{\alpha}}$ is a $\mathfrak{P}$-basis of $\omega$. Let $A$ be an arbitrary module. The projectivity of $\omega_{\alpha}, \alpha \in M$ implies the existence of exact sequences $0 \rightarrow K_{\alpha} \stackrel{i_{\alpha}}{\rightarrow} P_{\alpha} \stackrel{\pi_{\alpha}}{\rightarrow} A \rightarrow 0$ with $i_{\alpha} \in \mathfrak{H}_{\omega_{\alpha}}$ and $P_{\alpha} \in \mathfrak{P}_{\omega_{\alpha}}$. For $P=\sum_{\alpha \in M} P_{\alpha}$ let us define a mapping $\pi: P \rightarrow A$ by the formula $\pi\left(\left\{p_{\alpha}\right\}_{\alpha \in M}\right)=\sum_{\alpha \in M} \pi_{\alpha}\left(p_{\alpha}\right)$ (this can be done since only a finite number of $p_{\alpha}^{\prime \prime}$ s is non-zero). It is not too hard to show that $\pi$ is a homomorphism and, moreover, it is an epimorphism since $\pi_{\alpha}, \alpha \in M$ are. Let us introduce the following notation: $\iota_{\alpha}$ is the canonical embedding of $P_{\alpha}$ into $P, K=\operatorname{Ker} \pi$ and $i$ is the natural embedding of $K$ into $P$. Since $\bigcup_{\alpha \in M} \mathfrak{P}_{\omega_{\alpha}}$ is a $\mathfrak{P}$-basis of $\omega$ we have $P \in \mathfrak{P}_{\omega}$ by $(1,5)$ from [1] so that it suffices to show that $i \in \mathfrak{H}_{\omega}$. Let $P^{\prime} \in \bigcup_{\alpha \in M} \mathfrak{P}_{\omega_{\alpha}}$ be an arbitrary module and $\varphi \in \operatorname{Hom}\left(P^{\prime}, A\right)$ an arbitrary element. Then $P^{\prime} \in \mathfrak{P}_{\omega_{\alpha}}$ for some $\alpha \in M$ so that there exists a homomorphism 
$\psi^{\prime}: P^{\prime} \rightarrow P_{\alpha}$ with $\pi_{\alpha} \psi^{\prime}=\varphi$ (since $\left.i_{\alpha} \in \mathfrak{H}_{\omega_{\alpha}}\right)$. Putting $\psi=\iota_{\alpha} \psi^{\prime}: P^{\prime} \rightarrow P$ we have $\pi \psi=\pi \iota_{\alpha} \psi^{\prime}=\pi_{\alpha} \psi^{\prime}=\varphi$, which completes the proof.

Now we shall present two theorems concerning $\mathscr{E}$-purity.

Definition 3. We shall say that a projectively closed purity $\omega$ is $\mathscr{E}$-purity if it has a $\mathfrak{P}$-basis $\mathfrak{M}$ containing only cyclical modules.

Recall that a purity $\omega$ is called cyclically projective if to any module $A$ there exists an exact sequence $0 \rightarrow K \stackrel{i}{\rightarrow} P \rightarrow A \rightarrow 0$ where $i \in \mathfrak{H}_{\omega}$ and $P$ is a direct sum of cyclic $\omega$-projective modules.

Theorem 8. A projectively closed purity $\omega$ is $\mathscr{E}$-purity if and only if it is cyclically projective.

Proof. Firstly, let $\omega$ be an $\mathscr{E}$-purity and let $\mathfrak{M}$ be its $\mathfrak{P}$-basis containing only cyclical modules. Without loss of generality we can assume that $\mathfrak{M}$ is a set (in the opposite case take pair-wise non-isomorphic modules from $\mathfrak{M}$ ). Now the proof runs on the same lines as that of Theorem 3,4 and therefore we omit it.

Conversely, let us suppose that $\omega$ is cyclically projective and let us denote by $\mathfrak{M}$ the class of all cyclic modules from $\mathfrak{P}_{\omega}^{\prime}$. The inclusion $\mathfrak{M} \subseteq \mathfrak{P}_{\omega}$ gives $\mathfrak{H}_{\omega} \subseteq \mathfrak{S}^{\mathfrak{M}}$. On the other hand, to any module $A \in \mathfrak{P}_{\omega}$ there exists an exact sequence $0 \rightarrow K \stackrel{i}{\rightarrow} P \rightarrow$ $\rightarrow A \rightarrow 0$ where $i \in \mathfrak{H}_{\omega}$ and $P$ is a direct sum of cyclic modules from $\mathfrak{P}_{\omega}$, i.e. the modules from $\mathfrak{M}$. From $i \in \mathfrak{H}_{\omega}$ it follows that $A$ is isomorphic to a direct summand of $P$ so that $A \in \mathfrak{P}_{\omega} \mathfrak{M}$ by $(1,5)$ from [1]. Hence $\mathfrak{P}_{\omega} \subseteq \mathfrak{P}_{\omega}^{\mathfrak{M}}$ from which $\mathfrak{H}^{\mathfrak{M}} \subseteq \mathfrak{H}_{\omega}$ and the proof is complete. Theorem 9. For any $\mathscr{E}$-purity, the direct sum $A=\sum_{\alpha \in \Omega} A_{\alpha}$ is $\mathscr{E}$-flat if and only if any
module $A_{\alpha}, \alpha \in \Omega$ is $\mathscr{E}$-flat.

Proof. It suffices to use Theorem 2 since any cyclic module is compact.

\section{INJECTIVELY CLOSED PURITIES}

First of all we shall repeat some definitions. A module $Q$ is called injective with respect to a monomorphism $i: A \rightarrow B$ if for any diagram

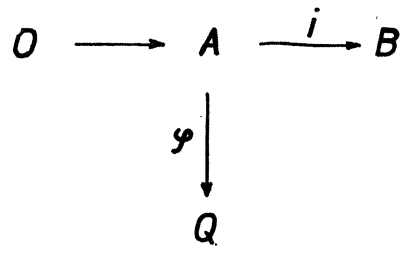


there exists a homomorphism $\psi: B-Q$ making the diagram

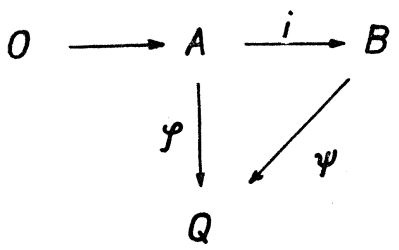

commutative. For a purity $\omega$ let us call a module $Q \omega$-injective if it is injective with respect to any $i \in \mathfrak{H}_{\omega}$. The class of all $\omega$-injective modules is denoted by $\mathfrak{Q}_{\omega}$. If $\mathfrak{M}$ is an arbitrary class of modules then the class $\mathfrak{H}_{\mathfrak{M}}$ of all monomorphisms $i$ such that any $M \in \mathfrak{M}$ is injective with respect to $i$, defines a purity (see $(1,16)$ in $[1])$, which we denote by $\omega_{\mathfrak{M}}$. The purity $\underline{\omega}=\omega_{\mathfrak{⿰} \mathfrak{Q}_{\omega}}$ is called the injective closure of $\omega$. Finally, a purity $\omega$ is called injectively closed, if $\omega=\underline{\omega}$, and a purity $\omega$ is called injective if to any module $A$ there exists an exact sequence $0 \rightarrow A \stackrel{i}{\rightarrow} Q$ with $i \in \mathfrak{H}_{\omega}$ and $Q \in \mathfrak{Q}_{\omega}$.

Theorem 10. A purity $\omega$ is injectively closed if and only if it is of the from $\omega_{\mathfrak{M}}$ for some $\mathfrak{M}$ of modules.

Proof. For an injectively closed purity $\omega$ we have $\omega=\omega_{\mathfrak{Q}_{\omega}}$. On the other hand we clearly have $\mathfrak{H}_{\mathfrak{M}} \subseteq \mathfrak{H}_{\mathfrak{Q}_{\omega_{\mathfrak{M}}}}$ while the converse inclusion follows at once from $\mathfrak{M} \subseteq \mathfrak{Q}_{\omega_{\mathfrak{M}}}$.

Theorem 11. Any injectively closed purity $\omega$ is bi-triangular.

Proof. In view of Theorem 10 and $(1,16)$ from [1] it suffices to show that $\omega$ is co-triangular. Let us consider the commutative diagram

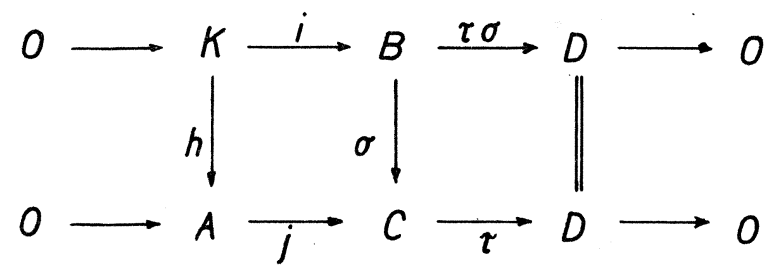

with exact rows where $\tau$ and $\sigma$ are given homomorphisms and $i \in \mathfrak{H}_{\omega}$. The existence of $h$ is guaranteed by $A \cong \operatorname{Ker} \tau$ and $\tau(\sigma i)=0$. We are going to show that $j \in \mathfrak{H}_{\omega}$. Let $V \in \mathfrak{Q}_{\omega}$ be an arbitrary module, $Q$ an arbitrary injective module containing $V$, 
$\lambda: V \rightarrow Q$ the canonical embedding, $\pi: Q \rightarrow Q / V$ the canonical projection and $\varphi: A \rightarrow V$ an arbitrary homomorphism. Let us consider the following diagram:

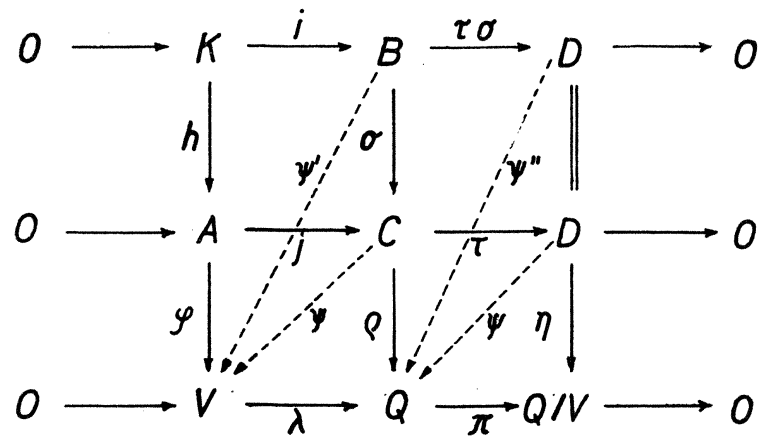

The existence of $\varrho$ satisfying $\varrho j=\lambda \varphi$ follows from the injectivity of $Q$ while the existence of $\eta$ satisfying $\eta \tau=\pi \varrho$ follows from $\pi \varrho j=\pi \lambda \varphi=0$. Therefore the diagram (4) (with full lines only) is a commutative diagram with exact rows. From $i \in \mathfrak{S}_{\omega}$ the existence of a homomorphism $\psi^{\prime}: B \rightarrow V$ with $\varphi h=\psi^{\prime} i$ follows. Further, $\left(\varrho \sigma-\lambda \psi^{\prime}\right) i=\varrho \sigma i-\lambda \varphi h=0$ implies the existence of a homomorphism $\psi^{\prime \prime}: D \rightarrow Q$ with $\psi^{\prime \prime} \tau \sigma=\varrho \sigma-\lambda \psi^{\prime}$. We have $\pi \psi^{\prime \prime} \tau \sigma=\pi \varrho \sigma-\pi \lambda \psi^{\prime}=\eta \tau \sigma$, so that $\pi \psi^{\prime \prime}=\eta$ since $\tau \sigma$ is an epimorphism. Further, from $\pi\left(\varrho-\psi^{\prime \prime} \tau\right)=\pi \varrho-\eta \tau=0$ we obtain $\lambda \psi=\varrho-\psi^{\prime \prime} \tau$ for a homomorphism $\psi: C \rightarrow V$. Finally $\lambda \psi j=\varrho j-\tau j=\lambda \varphi$ yields $\psi j=\varphi$ since $\lambda$ is a monomorphism and the proof is therefore complete.

Theorem 12. For an injectively closed purity $\omega$ the class $\mathfrak{H}_{\omega}$ is closed under taking direct sums.

Proof. Let $i_{\alpha}: A_{\alpha} \rightarrow B_{\alpha}, \alpha \in \Omega$ be an arbitrary set of elements of $\mathfrak{H}_{\omega}$. For any $\alpha \in \Omega$ we have a commutative diagram

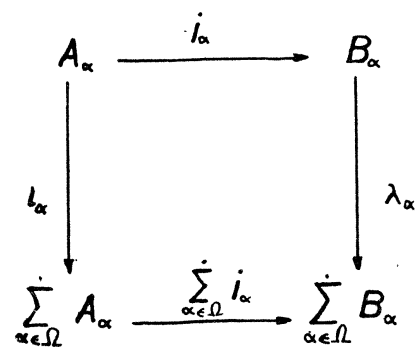

where $\iota_{\alpha}, \lambda_{\alpha}$ are canonical embeddings. For any $V \in \mathfrak{Q}_{\omega}$ and any $\varphi: \sum_{\alpha \in \Omega} A_{\alpha} \rightarrow V$ there exist homomorphisms $\psi_{\alpha}: B_{\alpha} \rightarrow V$ with $\psi_{\alpha} i_{\alpha}=\varphi \iota_{\alpha}\left(\right.$ since $\left.i_{\alpha} \in \mathfrak{S}_{\omega}\right)$. The universality 
of direct sums yields the homomorphism $\psi: \sum_{\alpha \in \Omega} B_{\alpha} \rightarrow V$ with $\psi \lambda_{\alpha}=\psi_{\alpha}$. Finally, from $\varphi \iota_{\alpha}=\psi_{\alpha} i_{\alpha}=\psi \lambda_{\alpha} i_{\alpha}=\psi\left(\sum_{\alpha \in \Omega} i_{\alpha}\right) \iota_{\alpha}$ and from the universality of direct sums (for $\sum_{\alpha \in \Omega} A_{\alpha}$ ) we get $\psi\left(\sum_{\alpha \in \Omega} i_{\alpha}\right)=\varphi$ and the proof is complete.

Theorem 13. Let $\omega$ be an injectively closed purity. Then the direct sum $A=\sum_{\alpha \in \Omega} A_{\alpha}$ is $\omega$-flat if and only if any $A_{\alpha}, \alpha \in \Omega$ is $\omega$-flat.

Proof. Theorem 13 follows from Theorem 12 in a similar way as Theorem 2 follows from Theorem 1 .

Theorem 14. Let $\omega$ be an injectively closed purity. Then the following three properties of a module $Q$ are equivalent:

1) $Q$ is $\omega$-flat,

2) $\left.\operatorname{Ext}(Q, V)=0^{2}\right)$ for any $V \in \mathfrak{Q}_{\omega}$,

3) for any $V \in \mathfrak{Q}_{\omega}, Q$ is co-projective with respect to the canonical embedding $\left.V \rightarrow \widehat{V}^{3}\right)$.

Proof. 1) $\Rightarrow 2)$ : If $Q$ is $\omega$-flat then $\left.\omega \operatorname{Ext}(Q, X)={ }^{4}\right) \operatorname{Ext}(Q, X)$ for any module $X$. For any $V \in \mathfrak{Q}_{\omega}$ we have $\omega \operatorname{Ext}(Q, V)=0$ so that 2$)$ is true.

2) $\Rightarrow 3$ ): From the exact sequence $0 \rightarrow V \rightarrow \hat{V} \rightarrow \hat{V} \mid V \rightarrow 0$ we obtain the exact sequence $\operatorname{Hom}(Q, \hat{V}) \rightarrow \operatorname{Hom}(Q, \hat{V} / V) \rightarrow \operatorname{Ext}(Q, V)$ which yields 3$)$.

3) $\Rightarrow 1$ ): To the module $Q$ let us choose an exact sequence $0 \rightarrow U \stackrel{j}{\rightarrow} F \stackrel{\eta}{\rightarrow} Q \rightarrow 0$ where $F$ is free and let us consider the diagram

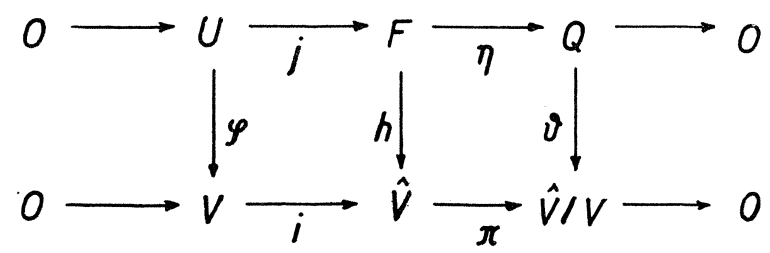

where $\varphi: U \rightarrow V$ is a given homomorphism. Since $\hat{V}$ is injective, there exists $h: F \rightarrow \hat{V}$ with $h j=i \varphi$. From $\pi h j=\pi i \varphi=0$ it follows that $\vartheta \eta=\pi h$ for some $\vartheta: Q \rightarrow \hat{\nabla} / V$ so that the diagram (6) is a commutative diagram with exact rows. By hypothesis 3 ) there exists a homomorphism $\psi^{\prime}: Q \rightarrow \hat{V}$ with $\pi \psi^{\prime}=\vartheta$. From $\pi\left(h-\psi^{\prime} \eta\right)=\pi h-$ $-\vartheta \eta=0$ we get $h-\psi^{\prime} \eta=i \psi$ for some $\psi: F \rightarrow V$. Finally, $\psi j=\varphi$ since $i$ is a monomorphism and $i \psi j=h j-\psi^{\prime} \eta j=i \varphi$.

\footnotetext{
${ }^{2}$ ) In this paper we shall write simply $\operatorname{Ext}(B, A)$ instead of $\operatorname{Ext}{ }_{\Lambda}^{1}(B, A)$.

3) $\hat{V}$ denotes the injective closure of $V$.

4) $\omega \operatorname{Ext}(B, A)$ is the subset of $\operatorname{Ext}(B, A)$ formed by all the sequences $0 \rightarrow A \stackrel{i}{\rightarrow} X \rightarrow B \rightarrow 0$ with $i \in \mathfrak{S}_{\boldsymbol{\omega}}$.
} 
Definition 4. We shall say that a class $\mathfrak{M}$ of modules is a basis of an injectively closed purity $\omega$, if $\omega=\omega_{\mathfrak{M}}$.

Now we shall formulate three theorems without proofs since they are dual to those of Theorems 3,4 and 7 respectively.

Theorem 15. If an injectively closed purity $\omega$ has a set as a basis then it also has a basis containing exactly one element.

Theorem 16. If an injectively closed purity $\omega$ has a set as a basis then it is injective.

Theorem 17. The intersection of any set of injective purities is an injective purity.

\section{3. $\mathscr{E}$-DIVISIBLE MODULES}

It is a well-known fact in the Abelian groups theory that a group $D$ is divisible if and only if it contains no maximal proper subgroups. This section is devoted to a generalization of this fact.

Recall that a module $D$ is $\omega$-divisible ( $\omega$ is any purity) if it is $\omega$-pure in any its extension ( $A$ is $\omega$-pure in $B$ if the canonical embedding $i: A \rightarrow B$ lies in $\mathfrak{H}_{\omega}$ ).

Throughout this section let $\mathscr{E}=\{\Lambda \mu, \mu \in M\}$ with $M \subseteq \Lambda$ be any set of maximal principal left ideals of $\Lambda$ satisfying $\Lambda \mu \subseteq \mu \Lambda$.

Definition 5. We shall say that a submodule $B$ of a module $A$ is an $\mathscr{E}$-submodule if the order of any non-zero element of $A / B$ belongs to $\mathscr{E}$. Further, we shall say that $B$ is an $\mathscr{E}$-maximal submodule of $A$ if $B$ is an $\mathscr{E}$-submodule of $A$ and it is maximal in $A$.

Theorem 18. If a module $D$ contains no proper $\mathscr{E}$-maximal submodule then $D$ is $\mathscr{E}$-divisible.

Proof. Let us suppose to the contrary that $D$ is not $\mathscr{E}$-divisible. By (1.53) from [1] there exist $\mu \in M$ and $d \in D$ such that $d \notin \mu D$. From this and from $\Lambda \mu \subseteq \mu \Lambda$ it follows $d \notin \Lambda \mu D$ and hence $D / \Lambda \mu D \neq 0$. Further, from the inclusion $\Lambda \mu \subseteq \mu \Lambda$ it easily follows that $\mu \Lambda$ is a left ideal of $\Lambda$ and therefore $\Lambda \mu=\mu \Lambda, \Lambda \mu$ being maximal. It is easy to see that $\Lambda / \Lambda \mu$ is a division ring (= non-commutative field). The factormodule $D / \Lambda \mu D$ can be considered as a $\Lambda / \Lambda \mu$-module by defining $(\lambda+\Lambda \mu)(d+$ $+\Lambda \mu D)=\lambda d+\Lambda \mu D$. By the well-known theorem on modules over a division ring (see e.g. [7]) the $\Lambda / \Lambda \mu$-module $D / \Lambda \mu D$ is completely decomposable. Therefore it contains a $\Lambda \mid \Lambda \mu$-submodule $D^{\prime} \mid \Lambda \mu D$ with $D / \Lambda \mu D / D^{\prime} \mid \Lambda \mu D \cong \Lambda / \Lambda \mu$. It is not too hard to show that $D^{\prime}$ is $\Lambda$-submodule of $D$. Considering $D, D^{\prime}, \Lambda \mu D$ as $\Lambda$-modules, we have $D / \Lambda \mu D / D^{\prime} \mid \Lambda \mu D \cong D / D^{\prime} \cong \Lambda / \Lambda \mu$. This implies that $D^{\prime}$ is an $\mathscr{E}$-maximal submodule of $D$ - a contradiction proving our theorem.

The following example shows that the converse of the preceding theorem does not hold in general. 
Example. As the ring $\Lambda$ we take the direct sum $\Lambda=C_{2}+C_{2}+C_{3}$ where $C_{2}$ and $C_{3}$ are prime fields of the characteristic 2 and 3 respectively. The ideal $C_{2}+C_{3}$ generated by $\mu=(0,1,1)$ satisfies all the conditions for the system $\mathscr{E}$. Direct calculation gives that $d=(0,1,0)$ is the only element from $D=C_{2}+C_{2}$ satisfying $\left.(0: \mu) \subseteq(0: d)^{5}\right)$. By (1.53) from [1] $D$ is $\mathscr{E}$-divisible since $d=\mu d$. On the other hand, the second direct summand $C_{2}$ is the maximal submodule of $D$ and it is easy to see that the order of the only non-zero element of $D / C_{2}$ is just $\Lambda \mu$.

Let us denote by $N=\bigvee_{\mu \in M}(0: \mu)$ the left ideal of $\Lambda$ generated by all the ideals $(0: \mu)$, $\mu \in M$.

Theorem 19. Let $D$ be an $\mathscr{E}$-divisible module satisfying $N \subseteq(0: d)$ for any $d \in D$. Then D contains no proper $\mathscr{E}$-maximal submodules.

Proof. Let us suppose to the contrary that there exists a proper $\mathscr{E}$-maximal submodule $H$ of $D$. If $d \in D-H$ is an arbitrary element then by Definition 5 there exists $\mu \in M$ with $(H: d)=\Lambda \mu$. The $\mathscr{E}$-divisibility of $D$, the hypothesis of our theorem and (1.53) from [1] imply the existence of $d^{\prime} \in D$ with $d=\mu d^{\prime}$. Here $d^{\prime} \notin H$ since $d \notin H$. On the other hand, $d^{\prime}=h+\lambda d, \lambda \in \Lambda, h \in H, H$ being maximal in $D$. Therefore, $d=\mu d^{\prime}=\mu h+\mu \lambda q=\mu h+\lambda^{\prime} \mu d \in H$, (since $\Lambda \mu=\mu \Lambda$ ) which is a contradiction proving our theorem.

Theorem 20. Let $D$ be an $\mathscr{E}$-divisible module satisfying $N \subseteq(0: d)$ for any $d \in D$. Then any epimorphic image of $D$ is $\mathscr{E}$-divisible.

Proof. Let $\varphi: D \rightarrow D^{\prime}$ be an arbitrary epimorphism, $d^{\prime} \in D^{\prime}$ an arbitrary element and $d$ any inverse image of $d^{\prime}$ under $\varphi$. Then $\left(0: d^{\prime}\right)=(\operatorname{Ker} \varphi: d) \supseteq(0: d) \supseteq N$. By (1.53) from [1] we have $d \in \mu D$ for any $\mu \in M$, hence $d^{\prime} \in \mu D^{\prime}$ for any $\mu \in M$ and $D^{\prime}$ is $\mathscr{E}$-divisible by (1.53) from [1] again.

\section{References}

[1] А. П. Мишина, Л. А. Скорняков: Абелевы группы и модули, Москва 1969.

[2] L. Fuchs: Abelian groups, Budapest, 1958.

[3] S. MacLane: Гомология, Москва, 1966.

[4] C. P. Walker: Relative homological algebra and Abelian groups, Ill. J. Math. 10 (1966), 186-209.

[5] L. Bican: A remark on projectively closed purities (to appear in Czech. Math. J.).

[6] I. Kaplansky: Projective modules, Annals of Math., 68 (1968), 372-377.

[7] A. Kertész: Vorlesungen über Artinsche Ringe, Budapest, 1968.

Author's address: Praha 8 - Karlín, Sokolovská 83, ČSSR (Matematicko-fyzikální fakulta UK).

${ }^{5}$ ) Recall that if $B$ is a submodule and $\mathfrak{M}$ a subset of a module $A$ then $(B: \mathfrak{R})=\{\lambda \in \Lambda$; $\lambda x \in B$ for any $x \in \mathfrak{M}\}$. 